\title{
Conduite synchronisée d'ouvrages hydrauliques en série
}

\author{
R. Benedetto \\ E.D.F. - G.R.P.H. « Méditerranée» \\ 140, avenue Viton - B.P. 540 \\ 13401 Marseille Cedex 09
}

\section{Introduction}

E.D.F. dispose, dans la région Provence-Alpes-Côte d'Azur, d'un outil de production d'énergie hydraulique, le " Gisement Durance-Verdon » dont les performances cinétiques ont été considérablement améliorées par l'informatisation du système de conduite, ainsi que par la mise en œuvre d'un nouveau mode de fonctionnement hydraulique.

Il est désormais possible de démarrer et passer à pleine charge les 18 centrales composant cet outil de production en moins de $10 \mathrm{mn}$, alors qu'avec l'ancien mode de conduite dit "au fil de l'eau", l'opération prenait 24 heures. Pour arriver à une marche synchronisée sur un site hydraulique vaste et complexe, les problèmes majeurs ont été la maîtrise du transfert des masses d'eau dans les canaux, ainsi que la mise en œuvre d'une assistance informatique puissante à l'opérateur qui devait piloter l'ensemble.

\section{Le site hydraulique}

Le site hydraulique est composé de deux branches principales: la branche Durance et la branche Verdon.

La branche Durance comprend un réservoir de tête, Serre Ponçon, situé à $780 \mathrm{~m}$ d'altitude, d'une capacité d'un milliard de $\mathrm{m}^{3}$. Entre ce lac et l'étang de Berre, les eaux de la Durance sont acheminées à travers $250 \mathrm{~km}$ de canaux, de galeries et de petites retenues intermédiaires d'une capacité de quelques millions de mètres cubes. Sur ce parcours, 15 centrales hydroélectriques ont été construites entre 1906 et 1974.

La branche Verdon comprend également un réservoir de tête, Ste Croix, situé à $477 \mathrm{~m}$ d'altitude, d'une capacité de 300 millions de $\mathrm{m}^{3}$. Le Verdon est peu canalisé, ses gorges étroites faisant office de canaux naturels. Ses eaux traversent 3 centrales et 2 réservoirs de quelques millions de mètres cubes avant de rejoindre la Durance à Cadarache.

\section{Rappel d'un fonctionnement dit « au fil de l'eau »}

Dans un système de centrales enchaînées, exploitées au fil de l'eau, le démarrage de chacune est décalé par rapport à celle qui la précède d'un retard pur lié à la structure du canal qui les relie. Ce retard est un paramètre fixe pour chaque canal.

Le contrôle du transfert de l'eau se fait en général à partir d'une seule mesure : la hauteur d'eau à la chambre d'eau de la centrale aval. L'élévation du niveau indique l'arrivée de l'eau turbinée par la centrale amont. L'objectif de la régulation est de maintenir cette côte à une valeur proche du point de consigne par action sur la vannage des groupes turbo-alternateurs.

Pour les centrales enchaînées sur la Durance, la somme des retards purs entre Serre Ponçon et St Chamas est de $24 \mathrm{~h}$. Ce qui implique que le démarrage total des 15 centrales se trouvait échelonné sur $24 \mathrm{~h}$, avant la mise en œuvre du système actuel. 


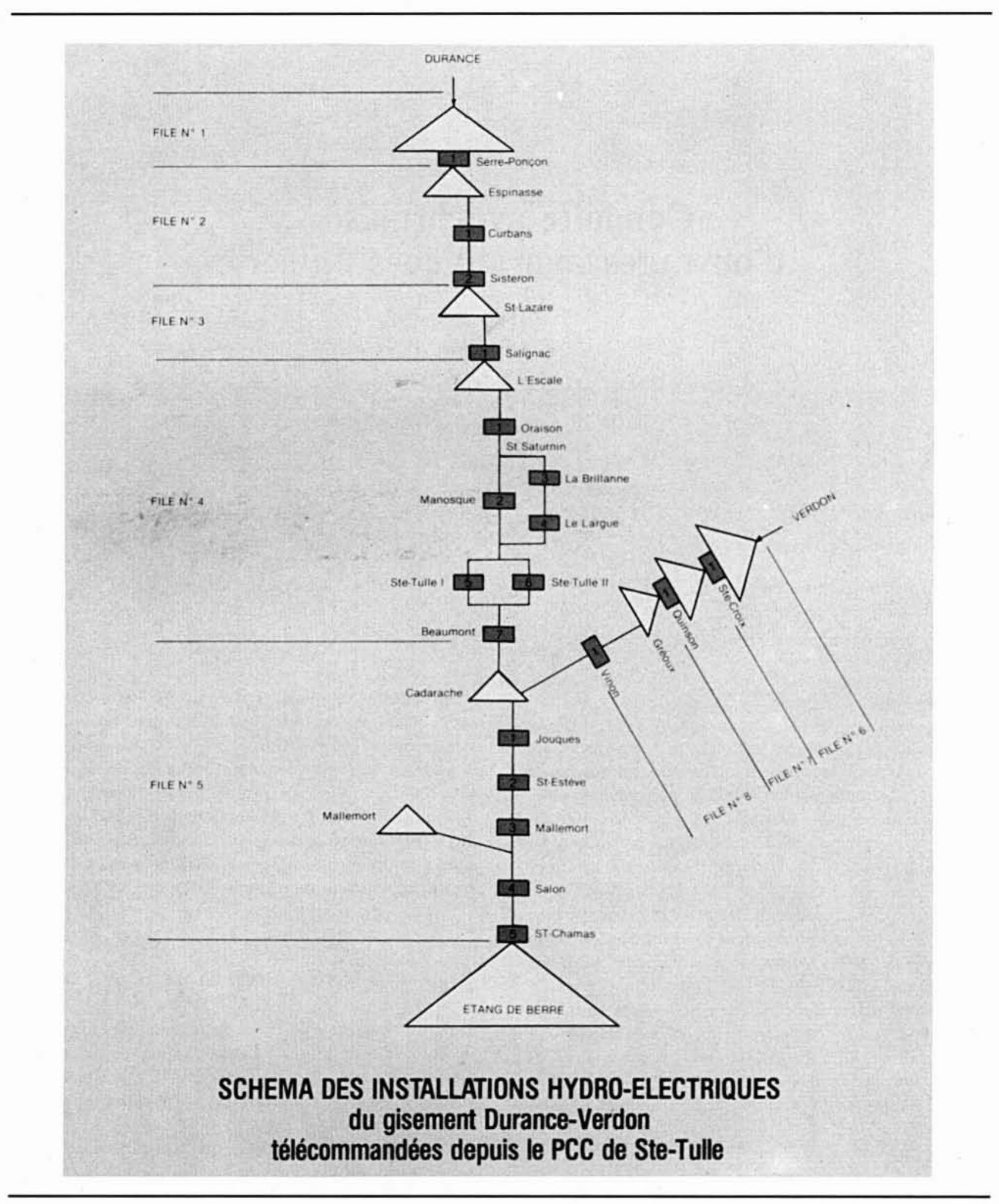

\section{Fonctionnement « synchrone » dit « à l'équivolume »}

Pour passer d'un système "fil de l'eau " à un système à démarrage synchronisé, il suffit en théorie d'envoyer au même instant un ordre de démarrage à toutes les centrales constituant la chaîne. Cela implique un changement total dans les méthodes de contrôle de transfert des masses d'eau, et a nécessité une étude préliminaire sur modèle mathématique, afin de quantifier les phénomènes hydrauliques qu'elle génère. Par ailleurs, le démarrage synchrone et le passage rapide à plein débit de deux centrales, reliées par des canaux qui n'ont aucune capacité de stockage, crée des perturbations importantes à l'amont et à l'aval de celles-ci. 
ègime transitoire

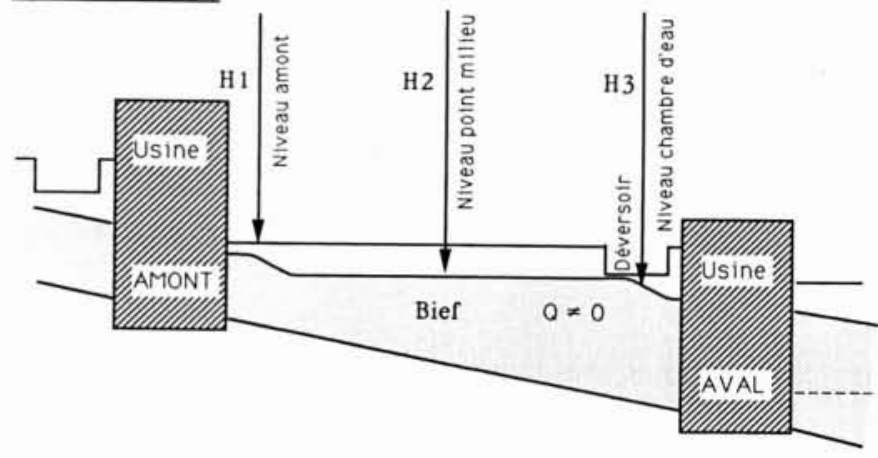

Régime établi

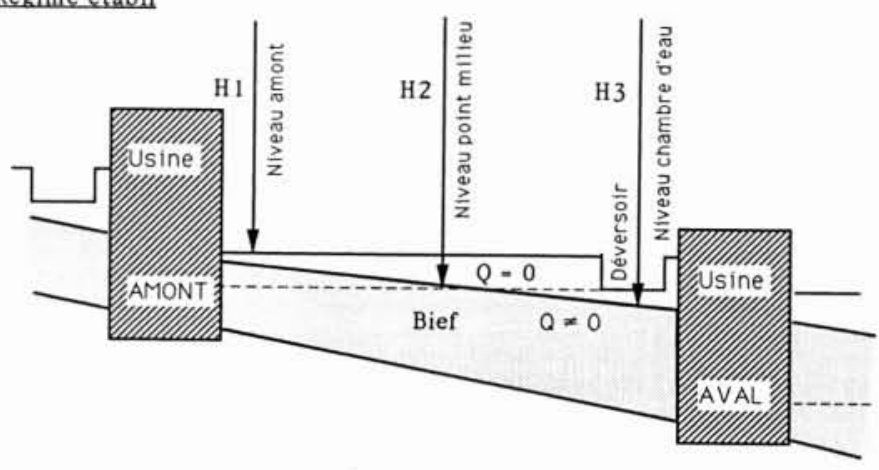

Elles se traduisent à l'aval immédiat de la centrale amont, par une élévation forte et rapide du plan d'eau, et à l'amont immédiat de la centrale aval, par une forte baisse du plan d'eau. La "bosse " d'eau ne doit pas dépasser le niveau des berges, sous peine de détériorer le génie civil. Le "creux" ne doit pas atteindre un niveau tel qu'il entraînerait le déclenchement des turbines. Au cours d'un arrêt synchrone, le phénomène est inversé.

On peut également constater qu'en théorie, si les démarrages sont parfaitement synchrones et les débits des deux centrales égaux, le volume d'eau contenu dans chaque tronçon de canal reste constant.

Il en résulte que pour obtenir un tel résultat de façon durable, il faut régler avec une très grande précision le débit des vannages. Les meilleures précisions obtenues par des mesures industrielles sont de 2 à $3 \%$, ce qui peut entraîner pour un débit de $300 \mathrm{~m}^{3} / \mathrm{s}$ et des erreurs de signe opposé sur les deux centrales d'un même canal, un écart de plus de $60000 \mathrm{~m}^{3}$ au bout d'une heure de fonctionnement. Compte tenu de la faible capacité utile des canaux de la Durance, cet écart deviendrait rapidement intolérable.

Le principe retenu a finalement été de remplacer la mesure du débit par celle du volume contenu dans le canal, puisqu'en définitive c'est cette valeur qu'il faut maintenir constante.

Or, s'il est aisé de mesurer le volume d'un canal à l'arrêt en connaissant sa géométrie, la surface du plan d'eau, et la hauteur de l'eau, la chose devient beaucoup plus délicate en régime transitoire lorsque le changement brutal et important des débits génère des ondes pouvant atteindre 2 mètres, crête à crête. Ce problème a été résolu par le Laboratoire national d'hydraulique de la Direction des Etudes et recherches d'E.D.F. Il a été découvert qu'un fonctionnement synchrone générait sur les canaux un point singulier particulièrement intéressant pour le calcul du volume. Ce point calculé sur modèle peut cependant être déterminé par l'observation des phénomènes physiques.

Au moment du démarrage synchrone, les deux centrales situées à chaque extrémité du canal, génèrent chacune une onde. Leur amplitude est proportionnelle à la variation de débit et leur signe opposé. En effet, sur un démarrage, l'onde amont est positive, l'onde aval est négative par rapport au niveau initial de la surface. Ces deux ondes se propagent le long du canal, l'une vers l'autre. Leur point de rencontre se situe, pour un canal donné, toujours au même endroit. En ce point, si les débits qui ont produit les deux ondes sont égaux, elles auront une amplitude égale, de signe opposé, elles s'annulent, et dans cette zone, la surface n'est pas perturbée.

Plus généralement, en régime établi, dans la mesure où le débit entrant demeure égal au débit sortant, la hauteur d'eau en ce point ne varie pas. Il se comporte comme un axe de basculement du plan d'eau, et on le nomme « milieu hydraulique". A partir de la cote en ce point, on peut obtenir une image précise du volume contenu.

Théoriquement, cette mesure serait suffisante pour déterminer le volume. En fait, en régime dynamique, elle ne permettrait pas d'anticiper les phénomènes transitoires. Le milieu hydraulique ne réagit à une variation qu'au bout d'un temps égal au 1/2 retard pur du canal, c'est-à-dire le temps mis par l'onde amont ou aval pour l'atteindre.

Afin de dynamiser le calcul, on a donc introduit la valeur des niveaux d'eau amont et aval du canal. Ceux-ci sont influencés immédiatement par une variation de débit. Il existe un autre intérêt à tripler les points de mesure. En cas de perte d'un capteur, il sera toujours possible de calculer d'une manière dégradée le volume. En pratique, le calcul peut être fait soit avec les 3 niveaux, avec uniquement le milieu hydraulique, avec le niveau amont et aval, ou avec le niveau aval seul à l'aide d'une fonction de transfert.

Dans le cas où l'on peut acquérir les 3 hauteurs d'eau, le principe de calcul du volume est le suivant :

Volume du canal $=\mathrm{S}_{1} \cdot \mathrm{H}_{1}$ niveau amont $+\mathrm{S}_{2} \cdot \mathrm{H}_{2}$ milieu hydraulique $+\mathrm{S}_{3} \cdot \mathrm{H}_{3}$ niveau aval.

$\mathrm{S}_{1}, \mathrm{~S}_{2}, \mathrm{~S}_{3}$, représentent les surfaces du canal pour lesquelles $\mathrm{H}_{1}, \mathrm{H}_{2}, \mathrm{H}_{3}$ sont représentatifs. Ces valeurs sont déterminées sur modèle pour chaque canal. 


\section{Régulation du volume du canal}

A partir du moment où le volume est connu, il suffit de mesurer l'écart par rapport à un volume de consigne et l'appliquer à un correcteur classique du type proportionnel intégral, fonctionnant avec comme unité le rapport du $\mathrm{m}^{3} / \mathrm{s}$ de débit au $\mathrm{m}^{3}$ d'écart de volume.

Cette correction pourra alors être appliquée sur le vannage grâce à un système d'asservissement, ce qui entraînera de légères variations autour du débit de consigne.

Afin que le système fonctionne correctement, il faut également tenir compte des débits intermédiaires liés aux apports naturels ou aux prélèvements tout au long des canaux. Leurs valeurs ne peuvent pas toujours être mesurées (multiplication des points, absence de seuils de tarage). Par contre, elles devront être estimées ou calculées avec une précision suffisante, et être prises en compte dans le calcul de débit de consigne des centrales.

\section{Le système informatique de conduite}

Le passage du mode de conduite "fil de l'eau " au mode " synchrone » a entrainé la refonte complète des principes de commande à distance.

Deux questions se sont principalement posées :

* Doit-on conserver un système de commande point à point?

En sachant que le système complet, c'est-à-dire 18 centrales, devrait être conduit par un seul opérateur, et que l'objectif était de démarrer l'ensemble et le passer à pleine charge en 10 minutes, on a procédé à une simulation pour quantifier le nombre d'ordres élémentaires qu'il aurait fallu donner pour réaliser la manœuvre. Il en est ressorti que 600 ordres sont nécessaires, ce qui représente, pour l'opérateur, un ordre par seconde. Il s'est donc avéré qu'une sérieuse assistance informatique était nécessaire.

A partir de là, une deuxième question s'est posée :

* Doit-on bâtir un système centralisé autour d'un très gros calculateur central effectuant tous les calculs, dialoguant avec l'opérateur et envoyant des ordres élémentaires aux centrales, dans lesquelles se trouveraient des équipements simples, récepteurs d'ordres et émetteur de données en retour?

L'inconvénient majeur d'un pareil schéma réside évidemment dans le risque de la perte totale de la conduite du process en cas de défaillance du calculateur central. Il a été décidé de s'orienter vers un système décentralisé composé d'une chaîne d'automatismes en série, découpée en 3 niveaux hiérarchiques baptisés $1^{\mathrm{er}}, 2^{\mathrm{e}}$ et $3^{\mathrm{e}}$ rang. L'avantage d'un tel schéma étant de créer des points de repli, assurant une marche dégradée en cas de défaillance d'un des éléments du système.

Pour chacun des automates de $1^{\mathrm{er}}, 2^{\mathrm{e}}$ et $3^{\mathrm{c}}$ rang, il a été défini d'une manière très précise ce que devait être leur domaine d'action, et surtout leurs limites afin qu'il n'y ait ni redondance, ni vide aux frontières.
Le rôle de l'automate de $1^{\text {er }}$ rang est de démarrer ou arrêter un groupe turbo-alternateur en effectuant les différentes manœuvres, de l'arrêt complet au couplage sur le réseau et retour à l'arrêt, tout en le protégeant contre ses incidents propres ou ceux venant de l'environnement.

Le rôle de l'automate de $2^{\mathrm{e}}$ rang est de mémoriser pour l'ensemble de la centrale, un programme de production sur $24 \mathrm{~h}$, composé de 48 valeurs $1 / 2$ horaires, et de l'exécuter en donnant aux automates de $1^{\text {er }}$ rang les ordres de démarrage ou d'arrêt nécessaires, ainsi que les ordres de réglage des vannages. Il a en outre la charge du calcul du volume du canal situé en amont et de son maintien à une valeur constante. Il peut recevoir de l'automate de $3^{\mathrm{e}}$ rang des consignes de production non prévisionnelles, et doit alors mettre en œuvre tous les moyens dont il dispose pour les exécuter. En cas d'impossibilité, il doit informer immédiatement le $3^{\mathrm{e}}$ rang de la nature et de l'importance du problème.

Toutes les données qu'il recueille sur le fonctionnement de la centrale sont envoyées au $3^{\mathrm{e}}$ rang pour archivage et visualisation.

L'automate de $3^{\mathrm{c}}$ rang, a pour fonction essentielle d'être l'outil de dialogue avec l'opérateur d'une part, et avec les 18 calculateurs de centrale d'autre part. C'est lui qui, à partir d'ordres globaux donnés par l'opérateur, va démultiplier en ordres plus élémentaires, qu'il va distribuer vers les calculateurs de centrales. Il possède également des fonctions d'archivage, de calculs statistiques, effectue des bilans sur l'eau et l'énergie, et est connecté au calculateur du dispatching régional d'où il reçoit l'essentiel des ordres de production.

Cette assistance informatique puissante mise à disposition de l'opérateur, a permis de ramener de 600 à 8 le nombre d'ordres à donner pour démarrer et passer à pleine charge l'ensemble du gisement Durance-Verdon.

\section{Conclusion}

Durant les 10 années qui ont suivi sa mise en service, grâce à une structure informatique souple, le système a pu constamment évoluer. Pour améliorer ses performances d'une part, et pour s'adapter aux évolutions des structures et des méthodes d'exploitation d'autre part.

Dans le domaine de la production d'énergie, la souplesse et les qualités cinétiques du process sont telles, qu'il a été possible de réaliser des gradients de montée en puissance de l'ordre de $600 \mathrm{MW}$ à la minute.

Dans le domaine de la distribution d'eau, la commande synchronisée des vannages offre aujourd'hui un confort qui n'existait pas lors du fonctionnement « fil de l'eau ». Il est en effet possible de répondre instantanément à une demande de débit, quel que soit la distance qui sépare le point de prélèvement du réservoir principal. 